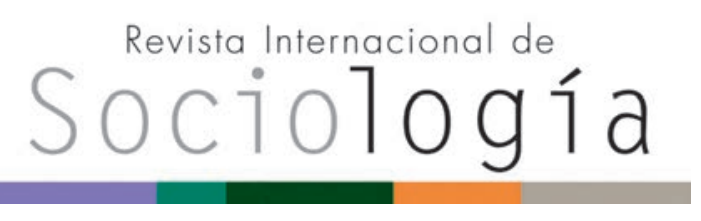

Revista Internacional de Sociología RIS vol. 77 (3), e132, julio-septiembre, 2019, ISSN-L:0034-9712 https://doi.org/10.3989/ris.2019.77.3.18.024

\section{IMPLICACIÓN PARENTAL, CAPITAL CULTURAL Y TRAYECTORIA FILIAL. PRÁCTICAS Y ESTRATEGIAS DISCURSIVAS}

\author{
Carlos Alonso Carmona \\ Universidad Pablo de Olavide \\ Calocar@upo.es \\ ORCID iD: https://orcid.org/0000-0003-3322-2307
}

\section{PARENTAL INVOLVEMENT, CULTURAL CAPITAL AND CHILDREN'S PERFORMANCE. PRACTICES AND DISCOURSIVE STRATEGIES}

Cómo citar este artículo / Citation: Alonso Carmona, C. 2019. "Implicación parental, capital cultural y trayectoria filial. Prácticas y estrategias discursivas". Revista Internacional de Sociología 77(3):e132. https://doi. org/10.3989/ris.2019.77.3.18.024
Copyright: ( 2019 CSIC. Este es un artículo de acceso abierto distribuido bajo los términos de la licencia de uso y distribución Creative Commons Reconocimiento 4.0 Internacional (CC BY 4.0).

Recibido: 15/02/2018. Aceptado: 30/11/2018 Publicado online: 24/09/2019

\section{Abstract}

We analyze family practices of parental involvement in children's schooling, through in-depth interviews and ethnographic observation. Instead of taking definitions from expert literature, we approach the concept of parent involvement from their own perspective. Results show that parental practices are conditioned by parents' cultural capital, available time and their children's prior academic achievement. Families strategically concep-tualise the meanings of involvement, so that they fit in the universe of possible practices. We conclude that parental involvement should be understood heeding the availability of resources, instead of the explanations based on familiar wishes.

\section{KEYWORDS}

Academic achievement; Discourse analysis; Familyschool relationships; Social class. 


\section{INTRODUCCIÓN}

En los últimos años, la implicación parental en lo escolar ha despertado gran interés entre las autoridades educativas. La idea de que unos vínculos fuertes entre familias y escuelas constituyen un factor de mejora educativa está presente en la legislación escolar de numerosos países (Egido, 2015) y ha ido ganando peso en España (Frías de Val, 2015). El profesorado comparte la idea de que el rendimiento diferencial del alumnado guarda mucha relación con la implicación parental, que suele entenderse como muestra del interés familiar por la escolaridad (Tarabini, 2015; Cabrera et al., 2011).

Académicos y decisores públicos han señalado que reforzar la implicación de las familias podría ser un remedio eficaz contra el fracaso escolar: habría que despertar el interés parental por los estudios y persuadir a los progenitores para que refuercen el acompañamiento escolar. Pero varios autores señalan que la inversión escolar parental depende de los recursos disponibles y el rendimiento filial previo. Mediante entrevistas en profundidad y observación etnográfica, analizamos la forma e intensidad de las estrategias escolares de 18 familias. Identificamos los factores que explican las principales diferencias en las prácticas. Después, analizamos la relación entre estas prácticas y las distintas posiciones discursivas. Finalmente, confrontamos nuestros resultados con el enfoque subyacente en numerosas propuestas de política educativa.

\section{MARCO TEÓRICO}

Pese a su reciente popularidad, el razonamiento que sitúa a la implicación parental como solución potencial- de los problemas educativos ha encontrado reticencias. Los estudios cuantitativos sobre su influencia en el rendimiento filial han llegado a resultados contradictorios. Muchos han estimado efectos de escasa magnitud, mientras que otros no encuentran correlación o sólo lo hacen bajo ciertas circunstancias. Estas contradicciones han abierto un amplio debate en la sociología de la educación norteamericana, centrado fundamentalmente en aspectos metodológicos (Abouchaar, 2016; Alonso Carmona, 2014).

Pero los argumentos más críticos han tomado habitualmente la forma de ensayos teóricos o investigaciones que emplean metodología cualitativa. La exponente más conocida de esta corriente es Annette Lareau $(2000,2011)$, quien argumenta: (1) las familias de distintas clases sociales cuentan con recursos desiguales para apoyar la trayectoria escolar filial; (2) esta diferencia de recursos condiciona distintos modos de practicar la implicación parental y (3) esta diversidad de prácticas se traduce en diferencias discursivas, la definición de lo que es un adecuado acompañamiento escolar varía con la clase social.
Numerosas investigaciones han puesto de relieve cómo la posición de clase condiciona el apoyo parental. La falta de tiempo y de conocimientos escolares restringe la capacidad de prestar ayuda directa o de evaluar las tareas en el hogar (Vincent y Maxwell, 2016). Muchas estrategias de inversión pedagógica requieren de recursos monetarios 0 , al menos, de cierta estabilidad económica (Cooper y Stewart, 2017). La relación con el profesorado está igualmente mediada por el estatus familiar: los estratos sociales privilegiados poseen mayor sintonía con las expectativas y el lenguaje docente (Reay, 2001; 1998), por lo que tienen más posibilidades de obtener lo que buscan cuando acuden a la escuela (McGhee y Schneider, 2009; McGrath y Kuriloff, 1999). En las clases trabajadoras, la distancia social al profesorado y la falta de familiaridad con el universo simbólico escolar pueden dificultar una relación fluida con el centro (Diamond y Gómez, 2004; Crozier, 1999).

Partiendo de estos hallazgos, varios análisis han acusado de sociocentrismo a los estudios tradicionales sobre implicación parental (Bower y Griffin, 2011; Auerbach, 2007). Estas aproximaciones suelen conceptualizar el apoyo parental a partir de las prácticas típicas de las clases medias, precisamente, las prácticas que las escuelas legitiman y valoran. Convertidas éstas en norma, se juzgan como deficitarios los esquemas de sentido y las estrategias habituales de las clases populares, estrategias muchas veces invisibles a los ojos de los docentes. Estas reflexiones tienen consecuencias metodológicas: las técnicas de recolección de datos se construirían para medir la implicación parental tal como la practican las clases privilegiadas (Prins y Toso, 2008). Sería este modo de relación familiar con la escolaridad el que las políticas públicas tratan de promocionar, sometiendo a las clases populares a un verdadero proceso de aculturación (Gillies, 2005) a la vez que se descarga sobre ellas la responsabilidad del fracaso escolar (Collet-Sabé et al., 2014).

Las familias tienen concepciones muy diversas del significado de "implicación". Éste depende de los recursos disponibles, por lo que tiende a adecuarse a las posibilidades objetivas. A esta diversidad de significados contribuye la propia ambigüedad del concepto, que engloba una serie muy amplia de estrategias y actitudes. Así, los progenitores menos familiarizados con el universo escolar priorizan en su definición el apoyo motivacional (Auerbach, 2007) o las estrategias de vigilancia y control (Lee y Bowen, 2006), prácticas que no requieren de un capital cultural específico. Reay (2005) muestra que las diferencias de clase en el apoyo a la escolaridad filial no son tanto de intensidad como cualitativas: las familias escogen los ámbitos y las prácticas de implicación con las que se sienten cómodas, en función de los recursos culturales y económicos disponibles. Con todo, 
muchos comportamientos de apoyo escolar apenas varían según la clase social (Martín Criado y Gómez Bueno, 2017b; Cooper y Crosnoe, 2007).

La implicación escolar no depende únicamente de los progenitores. Los propios hijos son parte activa de la relación entre familias y escuelas, y su comportamiento -en especial, su rendimiento académico- modifica las prácticas parentales. Las estrategias que los progenitores pueden emplear guardan mucha relación con la disposición de los niños y con su desempeño escolar. Quadlin (2015) muestra que muchas prácticas se intensifican o abandonan atendiendo a la trayectoria educativa filial y a los cambios que experimenta. Este tipo de hallazgos cuestionan la causalidad unidireccional, asumida por muchas investigaciones sociológicas y pedagógicas: habría que analizar las disposiciones familiares para explicar el rendimiento filial. Se ha probado igualmente que la trayectoria de los hijos modifica las expectativas parentales (Goldenberg et al., 2001), así como los estilos de autoridad en el hogar (Bronstein et al., 2005). De hecho, la reactividad de las prácticas familiares se ha presentado como explicación a las inconsistencias de la literatura sobre implicación familiar y rendimiento filial, anteriormente mencionadas. Los análisis, en especial los transversales, no recogerían el efecto benéfico de la implicación, porque muchas prácticas se emplean precisamente cuando aparecen dificultades (Domina, 2005). Además, hay abundantes evidencias de que el tipo y el grado de apoyo parental cambian con la edad de los hijos (Hill y Tyson, 2009).

En suma, las investigaciones precedentes señalan que las prácticas familiares de refuerzo pedagógico se adecúan fundamentalmente a las posibilidades efectivas, a los recursos utilizables y a la trayectoria filial. Aunque algunas hipótesis han postulado diferencias de valores entre clases sociales, hay fuertes indicios de que las clases subalternas han asumido plenamente la legitimidad del apoyo escolar (Martín Gimeno y Bruquetas Callejo; 2014, Lott, 2001) y lo practican en la medida de sus posibilidades (Martín Criado y Gómez Bueno, 2017b; Bennet, Lutz y Jayaram, 2012).

Ya hemos avanzado la naturaleza amplia y ambigua del concepto de implicación. Algunas investigaciones se ciñen en su definición a una serie - muy diversa- de prácticas, que incluirían, entre otras, la participación en órganos escolares, las reuniones frecuentes con el profesorado o la ayuda en las tareas en el hogar. Otros autores ensanchan su significado introduciendo actitudes ante la escolaridad, como la importancia concedida a los estudios o las expectativas académicas para los hijos. Varios van todavía más allá, incluyendo dentro de la misma categoría lo que son estrategias generales de crianza. Así, elementos como el sistema de sanciones en el hogar, las lógicas de autoridad familiar o la intensi- dad y el tipo de comunicación entre hijos y progenitores se han interpretado como distintas formas de implicación parental, aún cuando no guardan relación directa con el universo escolar. La diversidad en los procedimientos de operacionalización podría explicar los resultados contradictorios de las investigaciones cuantitativas: bajo la misma etiqueta, se estarían midiendo cosas distintas (Abouchaar, 2016).

Sin embargo, las diversas prácticas unificadas en la etiqueta de "implicación" resultan ser relativamente independientes unas de otras. Algunos estudios han advertido débiles o nulas correlaciones entre ellas. Para una misma unidad familiar, unos comportamientos no predicen los otros (Zellman y Waterman, 1998). También es muy discutida la relación entre ciertas actitudes - como las expectativas o los estilos parentales - y los comportamientos efectivos de apoyo escolar ${ }^{1}$ (Martín Criado y Gómez Bueno, 2017a; Llin Vañó et al., 2017; Hughes, Kwok y Hee, 2013). Esta hipertrofia conceptual parece responder al deseo de que los constructos metodológicos usados en las investigaciones cuantitativas incidan lo máximo posible en el rendimiento filial. Dándose por sentada la influencia positiva de la implicación parental en la trayectoria escolar de los hijos, el objetivo sería operacionalizar aquella, de forma que se aseguren correlaciones de suficiente magnitud. Toda disposición familiar que pueda tener un impacto estadístico en las calificaciones escolares es susceptible de ser asimilada a la categoría de "implicación". Ello no sólo ha alimentado la confusión metodológica, también ha desplazado a un segundo plano los razonamientos teóricos de causalidad. Se ha prestado mucha más atención a medir cuánto influye que a explicar cómo y por qué debería hacerlo (Zellman y Waterman, 1998:3).

La propuesta de este artículo es distinta. Asumiendo el carácter difuso del "apoyo parental a la escolaridad", nos proponemos analizar el concepto como categoría práctica. Dada su imprecisión y su legitimidad (como concepto incorporado al discurso especialista), la implicación parental se ha convertido en un objeto que se presta a todo tipo de apropiaciones e interpretaciones. Las familias maniobran discursivamente para validar y justificar sus estrategias, recurriendo a diversos - y cambiantes- argumentos, además de negociar constantemente los contenidos y significados de la implicación.

\section{Objetivos}

El objetivo del presente estudio es analizar las prácticas de apoyo parental a la escolaridad y los discursos en torno a las mismas, así como su variabilidad conforme a la clase social de pertenencia y la trayectoria de los hijos. Las investigaciones cualitativas sobre la cuestión han advertido diferencias en la forma en que las distintas clases practican 
la implicación escolar. Tales aproximaciones han construido un material teórico muy valioso, pero presentan, a nuestro juicio, dos déficits específicos que vamos a tratar de revisar: primero, han ignorado o subestimado el papel específico del rendimiento filial previo a la hora de entender las prácticas parentales. Segundo, habitualmente han analizado los discursos como simples descripciones de las prácticas, y no como jugadas estratégicas cuyo objetivo último es legitimarlas.

En cuanto al primer punto, ya se ha señalado que muchos comportamientos de apoyo escolar son reactivos. Las familias recurren a ellos en función de las dificultades que perciben en los hijos, por lo que la relación con la escolaridad difiere de forma importante, aun dentro de una misma clase social. La magnitud de los problemas escolares influye en la intensidad de la inversión parental y en el tipo de tácticas que pueden utilizarse.

En cuanto al segundo punto, entender los discursos como ilustración, más o menos imperfecta, de los comportamientos, es obviar la dimensión valorativa de las prácticas cotidianas (Martín Criado, 2014). Sobre la relación familiar con la escolaridad pesa toda una jerarquía simbólica de comportamientos aceptables e inaceptables. Los discursos familiares no sólo exponen las prácticas, también tratan de valorizarlas. De ahí que se utilicen estratégicamente, de cara a defender los comportamientos propios (insertos en una red de constricciones materiales y culturales) y criticar los ajenos.

\section{MÉTODO}

Esta investigación parte del análisis de datos cualitativos recogidos para la realización de una tesis doctoral en curso. El cuerpo principal de datos proviene de 18 entrevistas en profundidad realizadas a madres y padres con hijos cursando el primer año de Educación Secundaria Obligatoria (ESO) y residentes en la provincia de Sevilla. Las entrevistas fueron realizadas durante el primer trimestre del curso escolar 2016-2017. El contacto se produjo a través de tres institutos de educación secundaria, solicitando la participación voluntaria durante las reuniones iniciales con las familias del alumnado. Las entrevistas tuvieron una duración de entre 75 y 95 minutos.

Se procuró que la muestra fuese variada en tres aspectos: rendimiento filial previo, posición de clase y composición social de centro al que se acude. La primera cuestión era problemática, dado que el procedimiento de contacto (voluntario y a través de la propia institución escolar) conlleva un importante sesgo de selección. Se previó que los participantes serían progenitores con estrategias de crianza más pro-escolares que la media, y con hijos sin dificultades académicas graves. El primer supuesto no hemos podido comprobarlo (requeriría de la compa- ración con una muestra aleatoria), mientras que el segundo ha demostrado ser cierto sólo parcialmente. La gama de rendimientos filiales en las familias participantes era muy amplia, pero las conversaciones con los docentes indicaron que ninguno de los hijos pertenecía al "núcleo duro" de alumnos "disruptivos". Esto es, la muestra era variada en términos de rendimiento, pero mucho menos en comportamiento. Casi todas las participantes eran mujeres, cosa esperable dado que la labores de implicación escolar suelen recaer sobre las madres (Reay, 1998).

Para asegurar la diversidad de posiciones sociales, el procedimiento de contacto se realizó en tres centros distintos, todos de titularidad pública. La literatura ha teorizado un efecto centro en la configuración de la implicación parental, ligada en buena medida a las características socioeconómicas del alumnado (Desimone, 1999). Para examinar la interacción entre posición social familiar y composición social del centro, elegimos dos institutos con públicos antagónicos en términos de clase, además de uno de composición social mixta. El primero (1) de ellos es un instituto de reconocido prestigio a nivel local, cuyo público proviene mayoritariamente de las fracciones superiores de la clase media. El segundo (2) está situado en los límites de un distrito acomodado, en una zona fronteriza con varias barriadas obreras periféricas. La mayoría de su alumnado pertenece a las clases populares, aunque es también la opción de una minoría de familias de clase media. El tercer (3) centro está situado en un municipio tradicionalmente agrícola, a treinta kilómetros de la capital. Se encuentra en la zona más humilde de la localidad y casi la totalidad de su alumnado es de extracción obrera.

Para definir la posición de clase de los participantes nos apoyamos en las nociones de capital cultural, económico y simbólico de Pierre Bourdieu. En la teoría bourdieana, el espacio social es un espacio multidimensional. Además de la posición en las relaciones de producción económica, Bourdieu subraya la importancia de otros campos y sub-campos (especialmente el cultural) en la configuración de la estructura social (Baranger, 2004: 130). Esta pertenencia simultánea de los individuos a distintos campos jerarquizados difumina ocasionalmente las fronteras objetivas de clase. Con todo, y aun a riesgo de simplificar, se decidió emplear una categorización dicotómica, distinguiendo entre clase obrera y clase media, tal como proceden investigaciones similares (Lareau, 2000; Reay, 1998). En el primer grupo incluimos familias cuyos progenitores poseen estudios básicos o medios y que trabajan como asalariados en ocupaciones rutinarias de baja cualificación. En el segundo grupo se encuentran las familias cuyos responsables poseen estudios superiores y que trabajan en ocupaciones profesionales o tienen posiciones directivas. Pese a la clasificación binaria, el análisis tuvo en cuenta que ciertos atributos de clase se distribuyen gradualmente ${ }^{2}$. 
Tabla 1.

Entrevistados por origen social, sexo y rendimiento filial

\begin{tabular}{|c|c|c|c|c|c|}
\hline \multicolumn{6}{|c|}{ Clase obrera } \\
\hline IES & Nivel de estudios & Ocupación & Ocupación Cónyuge & Rendim. filial* & Sexo \\
\hline 2 & Primarios & Asistenta doméstica & Fontanero & Alto & M \\
\hline 2 & Incompletos & Ama de casa & Transportista & Medio & M \\
\hline 2 & Primarios & Asistenta doméstica & Dependiente & Bajo & M \\
\hline 2 & Primarios & Cocinera & Transportista & Alto & $M$ \\
\hline 2 & Medios (BUP) & Dependiente & Administrativa & Alto & $\mathrm{H}$ \\
\hline 2 & Medios (FP) & Cocinera & Comercial & Medio & M \\
\hline 2 & Medios (FP) & Ama de casa & Montador mecánico & Alto & M \\
\hline 3 & Primarios & Camarera & Jornalero & Medio & $M$ \\
\hline 3 & Primarios & Encuadernadora & Monoparental & Bajo & M \\
\hline \multicolumn{6}{|c|}{ Clase media } \\
\hline 1 & Superiores (Uni) & Prof. Universidad & Prof. Universidad & Alto & $M$ \\
\hline 1 & Superiores (FP) & Propietaria gestoría & Propietario gestoría & Alto & $M$ \\
\hline 1 & Superiores (Uni) & Ingeniera & Abogado & Bajo & M \\
\hline 1 & Superiores (Uni) & Biólogo & Ama de casa & Medio & $\mathrm{H}$ \\
\hline $1^{\text {** }}$ & Medios (BUP) & Propietario sector construcción & Ama de casa & Alto & $\mathrm{H}$ \\
\hline 1 & Superiores (Uni) & Prof. Secundaria & Monoparental & Medio & M \\
\hline 1 & Superiores (Uni) & Psicóloga & Periodista & Medio & M \\
\hline 2 & Superiores (Uni) & Ama de casa & Ingeniero & Alto & M \\
\hline 2 & Superiores (FP) & Delineante & Ama de casa & Bajo & $\mathrm{H}^{\star * *}$ \\
\hline
\end{tabular}

${ }^{*}$ Hemos distinguido entre rendimientos altos (suspensos inexistentes o muy ocasionales en primaria), medios (suspensos recurrentes pero sin dificultades para promocionar) y bajos (suspensos habituales con dificultades para promocionar o habiendo repetido ya un curso).

** Pese a no poseer estudios superiores, hemos incluido a este participante en clase media, dado su alto capital económico y su posición de propietario con asalariados.

${ }^{* * *}$ Ambos cónyuges estuvieron presentes en la entrevista, aunque con un papel protagonista del padre.

La investigación utiliza dos conjuntos adicionales de datos. Se realizaron cuatro observaciones etnográficas en la sala de profesores durante las reuniones de coordinación de tutores, cada una de una hora de duración. Se realizaron, además, 11 observaciones de reuniones entre tutores y algunas de las familias participantes. Estas últimas pudieron ser grabadas en audio y posteriormente transcritas. En nuestro país, el análisis de este material constituye una novedad en la investigación sociológica. Como veremos, suponen una fuente privilegiada para examinar los discursos parentales y cómo estos se negocian frente a los representantes del sistema escolar ${ }^{3}$. Estas reuniones tuvieron una duración de entre 15 y 50 minutos.

Como investigación que utiliza métodos cualitativos, las conclusiones extraídas de este análisis no pretenden alcanzar representatividad estadística, pero sí clarificar procesos de causalidad (Mitchell, 1983). El trabajo del investigador se centra en extraer conclusiones sobre el vínculo que une ciertos fenómenos y ofrecer un esquema explicativo. Siguiendo a Small (2008:8): "la potencia de la investigación cualitativa se basa en el entendimiento del por qué y del cómo, no del cuánto. Mejorar la validez de estos trabajos significa mejorar su capacidad para responder a estas preguntas".
El análisis de los datos se ha realizado entendiendo los discursos como jugadas estratégicas. Estrategias no sólo de presentación frente al interlocutor, también estrategias de negociación del sentido, tratando de redefinir los conceptos legítimos para adecuarlos a las prácticas propias. Esto conlleva la oposición a dos procedimientos habituales. Uno, el que asume que la implicación es un concepto estanco y, una vez definido éste con precisión, evalúa las prácticas parentales a partir de su mayor o menor correspondencia con el modelo ideal (Auerbach, 2007). Aquí defendemos la necesidad de entender las propias definiciones de los sujetos y la lógica que subyace a ellas, frente al uso de categorías preconstruidas (Martín Criado, 1991). Dos, las aproximaciones esencialistas, que entienden que a cada estrato social le corresponde un conjunto integrado y coherente de posiciones ante la escolaridad. El análisis mostrará que los sujetos alternan razonamientos muy diversos a lo largo del discurso, producto de la convivencia entre legitimidades contradictorias o del choque entre una legitimidad reconocida y una serie de prácticas incoherentes con la misma.

La presente investigación trata de demostrar que los discursos familiares no son simples exposiciones descriptivas que ilustran las prácticas posibles dadas unas condiciones objetivas. Con los discur- 
sos, los progenitores tratan de situarse simbólicamente en las posiciones legítimas: presentarse como adecuados criadores y demostrar pro-escolaridad. El objetivo de los argumentarios parentales es, fundamentalmente, salvar la cara frente a su interlocutor ${ }^{4}$ (Goffman, 1971).

\section{RESULTADOS}

Las 18 familias estudiadas presentan un amplio abanico, en tipo e intensidad, de prácticas de inversión pedagógica, por lo que las diferencias argumentativas son igualmente amplias. Empezaremos describiendo las diferencias de prácticas, para luego pasar a analizar los razonamientos que las validan y legitiman ante los otros. En el apartado de conclusiones justificaremos que esta aproximación a la relación familiar con la escuela nos lleva a conclusiones de interés para la investigación y la política educativa.

\section{Recursos disponibles y diversidad de prácticas}

Las prácticas familiares difieren no sólo en intensidad, también en las formas en que la implicación se practica y conceptualiza. Hemos identificado, en línea con los hallazgos de investigaciones precedentes, una serie de variables fundamentales para entender la naturaleza de estas prácticas: rendimiento filial, tiempo disponible y clase social de pertenencia -que influye, principal aunque no únicamente, a través del capital cultural-.

En primer lugar, existe una relación clara entre implicación parental y desempeño escolar previo de los hijos. Por varios motivos. Uno, buena parte de los comportamientos de apoyo son reactivos, las familias los despliegan cuando ven peligrar la trayectoria filial. Dos, en muchos casos la ayuda familiar se proporciona a voluntad de los propios hijos y su solicitud es más probable cuando empiezan a experimentar dificultades. Tres, cuando los hijos no la demandan, su disposición a aceptar la intervención paterna es muy variable y está igualmente relacionada con su grado de éxito escolar. Cuando la relación de los hijos con la institución deviene especialmente problemática, es habitual el boicot a las estrategias parentales. La continuada resistencia filial puede llevar a los padres a distanciarse del ámbito escolar o a elegir comportamientos menos invasivos, por ejemplo, limitándose al control de los horarios de estudio. Un hecho elocuente sobre la importancia del rendimiento filial para entender las prácticas parentales es la comparación entre hermanos. Dentro de una misma familia, el acompañamiento escolar es muy distinto entre ellos cuando las calificaciones son igualmente dispares:

Y es una cosa que ahora me pesa, me pesa porque creo que al chico lo he malacostumbrado, y se ha vuelto muy... dependiente. Porque claro, le cuesta. Yo le he ayudado mucho siempre, con sus deberes y tal, tardes casi enteras en la mesa del salón los dos. Pero la mayor, que nunca ha tenido problema, pues, ¿cómo te digo?, lo hace todo sola. Nunca ha necesitado ayuda, ella es muy... lo hace todo sola, vamos

(Mujer, clase media, rendimiento filial medio).

Podemos decir, grosso modo, que la intensidad de la inversión pedagógica es menor conforme los hijos van consolidado una trayectoria exitosa. En cambio, la intervención parental es más fuerte si las calificaciones filiales han sido inestables y, especialmente, cuando se dan empeoramientos repentinos importantes. Pero en una minoría de casos (que analizaremos con mayor detalle más adelante) la implicación puede declinar precisamente ante la constancia de un fracaso escolar irreversible. El hecho de que la inversión escolar sea mayor ante rendimientos intermedios, frente a los muy altos o muy bajos, ha sido advertido por otras investigaciones (Pérez Sánchez, Betancort Montesinos y Cabrera Rodríguez, 2014).

En segundo lugar, el tiempo disponible condiciona de forma evidente la capacidad familiar para prestar apoyo. Las prácticas que más dependen de éste - como la ayuda con los deberes - son más habituales cuando los progenitores tienen jornadas laborales más cortas, o en madres que se dedican al trabajo doméstico no remunerado en el propio hogar ${ }^{5}$. Tal disponibilidad de tiempo no es independiente del estatus socioeconómico, dado que las clases medias suelen tener horarios de trabajo más reducidos y flexibles, en comparación con sus homólogos de clase obrera (Prins y Toso, 2008). Otro aspecto, menos investigado, que constriñe el tiempo disponible es el número de hijos y la edad de los mismos. Un apoyo intensivo en tiempo es más difícil conforme aumenta el tamaño de la fratría, sobre todo si los hijos en edad escolar conviven con hermanos pequeños que requieren de cuidados continuos.

En tercer lugar, la clase social ha sido la variable protagónica de las investigaciones ${ }^{\circ}$ precedentes sobre la cuestión. Nuestros datos apuntan en el mismo sentido que ellas: confirmando análisis como los de Reay (2005), vemos que la implicación se practica -y se define- en términos más pedagógicos cuanto mayor es la afinidad familiar con el universo escolar. Tal variabilidad de concepciones no es la simple expresión de una diversidad de prácticas, sino que constituye toda una lucha en torno al verdadero significado de "implicarse". Las distintas clases sociales negocian el sentido de la implicación para adecuarla a los comportamientos posibles; por ejemplo, en clases populares, a los menos dependientes del capital cultural. En estos estratos, es habitual categorizar el apoyo parental como acompañamiento motivacional ("estar ahí, que vea que te tiene, decirle que es importante") o se exhibe el compromiso con la escolaridad a través de actividades "mundanas": 
He intentado implicarme un poquito para que ella viera que... verás, que me tiene ahí ¿sabes? Ella siempre... "y mi madre viene, si tu madre no puede, mi madre sí puede". Siempre, para eso sí, y si tienen que ir a la biblioteca que yo las llevo y las recojo. Y si le hace falta me pregunta "mamá, ¿podemos quedar aquí para un trabajo?" o "¿tú me puedes ayudar a buscarle la ropa a no sé quién...?" para cualquier actuación que ha tenido el cole... o "¿puedes maquillar a las niñas tú?". Yo para eso sí, y ella cuenta mucho conmigo porque los estudios son lo primero (Mujer, clase obrera, rendimiento filial alto).

Estas concepciones, que subrayan los aspectos moralizantes de la implicación, son muy diferentes de las que aparecen en las entrevistas con progenitores de clase media. En su relación con la escolaridad, estos recalcan los contenidos más propiamente didácticos, como tomar la lección ante un examen o la ayuda con las tareas. Las tutorías constituyen un espacio privilegiado para examinar estas diferencias. Durante ellas, las familias obreras muestran su compromiso de socializar a los hijos en actitudes pro-escolares y remarcan el control de horarios y las sanciones. Mientras, los padres de clase media comentan en mucha mayor medida el contenido de los temarios, los sistemas de calificación o aluden a aspectos concretos de aprendizaje. Frente a la actitud estimuladora y vigilante que tratan de mostrar las clases populares, los progenitores de estratos superiores se presentan como una suerte de enseñantes complementarios:

\section{Madre - No se puede decir que no nos impliquemos... es que en matemáticas yo le preparo exámenes, le he preparado el último trimestral y, bueno, al principio fallaba mucho, le hice un sistema de ejercicios, au- mentando poco a poco la dificultad, y al final le salian muy bien. Cuando me dijo: "pues mira, el examen me ha salido muy bien" y luego me dijo la nota, seis con siete, si yo recuerdo bien... (Tutoría, pareja de clase media, rendimiento filial bajo).}

La desigual disponibilidad de tiempo, capital cultural y notas filiales explican en buena medida la pluralidad de prácticas, que, a su vez, están asociadas a una diversidad de esquemas de sentido. Esto es, las representaciones de la implicación parental tienden a asimilarse a los comportamientos efectivos. En los siguientes apartados, vamos a examinar con más profundidad cómo los discursos familiares se usan para validar estas prácticas. Para ello debemos tener en cuenta esta variedad de interpretaciones, pero también el juego de legitimidades en torno al apoyo escolar y la crianza.

\section{Autonomía, confianza y control}

En los discursos familiares, la implicación parental colisiona a menudo con otro concepto legítimo, el de la autonomía infantil. La idea de que una buena crianza debe producir hijos resolutivos e independientes está muy presente en el imaginario parental, y ha sido alimentada por la pedagogía y demás especialistas de la infancia. El niño "mimado" y el padre "sobreprotector" constituyen los anti-sujetos por excelencia de muchos relatos familiares. Es fácil ver que los discursos sobre el apoyo parental (que dibujan una familia copartícipe y, a la postre, facilitadora de los retos escolares) pueden entrar en conflicto con la legitimidad del "hijo autónomo": las familias que apoyan se lo estarían poniendo sencillo a sus retoños. Un niño ayudado es un niño que invierte menor cantidad de esfuerzo, y a la larga, se acostumbraría "a la buena vida", quebrando la estructura familiar de derechos y deberes. Esto último podría ser incluso fuente de problemas escolares futuros, dado que la familia no puede acompañar indefinidamente al menor en su trayectoria educativa. Llegado el punto, los hijos fracasarían al verse privados del apoyo parental.

Esta tensión discursiva entre dos legitimidades discordantes (autonomía/implicación) es central para entender buena parte de las argumentaciones en torno a la relación con la escuela. Aquí encontramos, no obstante, diferencias de clase en la justificación simbólica de uno de los polos legítimos. Para las clases medias, más familiarizadas con el lenguaje pedagógico, la noción de autonomía infantil bebe, en mayor medida, del discurso especialista y su desaprobación de la dependencia filial. En las clases trabajadoras, esta legitimidad experta convive con -y se interpreta a través dela noción de una crianza "tradicional", más desapegada del ámbito escolar:

"Que hoy creo que ayudamos más con los hijos de lo que nuestros padres con nosotros, y estamos como más encima... pues creo que da pie a que haya gente que malentienda esa preocupación y al final se rompe esa autoridad de antes, que debe existir en la familia" (Hombre, clase obrera, rendimiento filial alto).

Los sujetos entrevistados se sitúan en distintas posiciones a lo largo del eje autonomía-implicación. La existencia de dos polos legítimos abre la posibilidad a todo tipo de jugadas discursivas para valorizar las prácticas propias. Frente a las familias que ayudan menos que nosotros, se invocan los argumentos pro-escolares que jerarquizan a las familias en función del apoyo en los estudios: los otros serían padres que no se preocupan por sus hijos. Frente las familias que ayudan más que nosotros se recurre a la tesis de la independencia filial y sus bondades. Esta última estrategia gira en torno a un concepto clave: la confianza.

Yo confío en ella, es ella la que tiene que estudiar... ahí saben con tiempo cuándo le ponen el examen (...) Si hay que echar una mano, se echa en lo que se puede, pero, o sea, yo tampoco tengo tiempo para controlarlo todo, tengo tres, todo el día para arriba, para abajo (Mujer, clase obrera, rendimiento filial alto). 
Los protagonistas de ambos fragmentos comparten algunas características comunes: tres hijos, trabajos a jornada completa (al igual que sus cónyuges), bajo capital cultural y buen rendimiento filial. Las dificultades de prestar apoyo directo y la percepción de que, en todo caso, no es necesario, les llevan a situarse en el polo ideal de la autonomía. El apoyo continuado a la escolaridad sería propio de progenitores imprudentes que no han inculcado responsabilidad a los hijos. Además, la implicación se presenta como remedio tardío y engañoso ante el bajo rendimiento, se estarían intentando paliar a destiempo las consecuencias de una socialización temprana defectuosa.

Estos informantes son los que más abiertamente cuestionan el nuevo modelo de relación intensiva con la escuela, y los que más aluden a la separación de competencias entre familias y docentes (Lareau, 2000). Pero esta distancia es presentada como lealtad hacia los valores escolares: la mejor muestra de implicación parental sería el reconocimiento de la profesionalidad y la autonomía del profesor.

En el extremo opuesto encontramos a madres de clase media con jornadas reducidas cuyos retoños experimentan graves dificultades. Bien armadas de tiempo y conocimientos, practican un apoyo intensivo en el hogar y contactan continuamente con el cuerpo docente. En estos casos se realiza una demostración constante de "patriotismo" escolar, acompañado de múltiples acusaciones a las familias no implicadas, hasta el punto de cargar sobre ellas la culpa de todos los problemas del sistema educativo:

\footnotetext{
$Y$ ya tiene que ser bastante duro (para un profesor) entrar día sí y día también en una clase, y que los niños te digan... cualquier cosa. Vamos, las cosas que se oyen son de traca. Falta educación, pero en los padres. Que los padres se creen que con once años, doce años, los niños ya son adultos, y venga, y todo vale. Y no hay implicación, pero la implicación falta en los padres. (Mujer, clase media, rendimiento filial bajo).
}

Pero la mayoría de las familias circulan de forma inestable entre ambos extremos. Dispuestos a cumplir los preceptos legítimos del apoyo parental, pero temerosos de ser acusados de sobreprotectores, su discurso oscila constantemente buscando "el justo punto medio". Así, el objetivo de los discursos es encajar este punto medio con las prácticas propias, negociando lo que es una adecuada inversión pedagógica, y manejando estratégicamente la legitimidad de la implicación y la confianza. Este proceso de negociación discursiva se intensifica en las reuniones de tutoría. Aquí es donde las familias enfrentan mayor presión para certificar su grado de seguimiento, especialmente cuando los niños tienen dificultades escolares:

Profesor - "Entonces eso lo tiene que tener ella en su cuaderno, y yo no lo he visto. Entonces claro, si ustedes no están al tanto de eso..."
Padre - A ver, insisto, yo sí le pregunto. No todos los días, pero cada dos o tres días. Tampoco le voy a preguntar todo el tiempo porque no quiero que la niña... vea que no confío, que no me fío de ella. Que eso, también, para su autoestima... claro... (Tutoría, pareja de clase media, rendimiento filial bajo).

Estas maniobras dialécticas son particularmente visibles cuando se habla del control y del apoyo a las tareas escolares que han de realizarse en el hogar. En torno a estas prácticas se expresa con más fuerza la tensión discursiva a la que estamos haciendo referencia. A la vez que se reprueba a los padres "desconectados", se marcan distancias con los que practican una ayuda demasiado intensa; la clásica figura del "padre que le hace los deberes al niño" sería la representación extrema de este comportamiento ilegítimo. Una forma bastante habitual de resolver esta tensión es valorar el grado de implicación en función de la edad filial. Dado que el apoyo parental suele decaer conforme los hijos crecen, las familias han experimentado distintos momentos en su grado de inversión a lo largo de la edad escolar de los hijos. Así, se seleccionan tácticamente las distintas fases de la trayectoria filial para satisfacer los dos postulados contradictorios. El apoyo más intenso, característico de los primeros años, demostraría el interés por la escolaridad. Con el avance de los cursos, las familias se distancian paulatinamente de la vida escolar (a distintos ritmos en función de los recursos parentales y las calificaciones filiales) y recurren a la noción de autonomía:

Porque tenemos unos amigos que se ponen a estudiar con él. Y de eso no se trata. Vale, que eso se hace hasta... yo que sé, primero de ESO. Después llegan cursos que tú no eres capaz de seguirle, y... ¿qué haces? ¿Lo dejas tirado al chaval, lo dejas tirado porque no le has enseñado a estudiar? (Hombre, clase media, rendimiento filial alto).

\section{Descomponiendo la implicación parental}

Como hemos visto, la implicación es un concepto amplio y complejo, que reúne en su seno una multitud de prácticas no necesariamente relacionadas. Estas características facilitan que las familias negocien el sentido de la misma, definiéndola a conveniencia hasta adecuarla a las prácticas propias. Jugando con la ambigüedad del término, los progenitores "trocean" la categoría de implicación y valoran independientemente cada uno de los elementos que forman parte de ella. Esta parcelación conceptual posibilita la convivencia de las dos legitimidades antes señaladas. Desplegado el abanico de prácticas posibles, se enfatizan aquellas que se emplean con mayor frecuencia para resaltar la preocupación por los asuntos escolares. Por su parte, aquellos ámbitos en los que no se interviene darían cuenta del compromiso paterno con la independencia filial, y funcionan como muestrario de una responsabilidad ya inculcada. 
Los ejemplos de este procedimiento son abundantes en los discursos familiares. Una separación habitual es la que se realiza entre la implicación parental en el hogar y en la escuela. Cuando el seguimiento a la escolaridad en el hogar es continuado pero hay poco contacto con el cuerpo docente, esta última práctica es señalada como superflua y poco recomendable. En estos casos, los progenitores comentan orgullosamente que "a ellos no les llaman"; las tutorías serían un espacio de reprobación docente a la mala praxis parental. Además, quienes buscan información en los docentes serían aquellos padres que no pueden obtenerla de los hijos, aquellos que no han sido capaces de forjar relaciones de confianza con ellos -en línea con el nuevo modelo legítimo de crianza- (Martín Criado et al., 2000). Cuando la situación es la contraria (se consulta frecuentemente al profesor, pero hay poca ayuda efectiva en casa) los argumentos se invierten igualmente. Para hablar del apoyo a las tareas se utilizan los discursos favorables a la autonomía infantil. A la vez, se pone en valor la presencia física en el centro y se manejan todos los alegatos sobre las bondades de la relación familia-escuela. Acudir a las tutorías sería el mejor exponente de esta relación, su representación arquetípica. Esto implica defenderse de las acusaciones que relacionan contacto frecuente con problemas escolares filiales:

\footnotetext{
Yo suelo ir. No soy yo de dejarlas... al instituto ya he ido, vamos (el curso acaba de empezar), he pedido tutoría y ya he ido vamos. Y me dijo la profesora: "¿es por algo en particular?" y le digo: no, que a mí me gusta hablar con los profesores para saber cómo va mi hija, cada cierto tiempo me gusta ir, siempre, en ese aspecto sí estoy encima de ella, aunque fuese bien, que no hace falta que vaya malamente para decir "tú, hija, ¿qué?", vaya bien o mal me gusta ir a hablar por... para que vea que estoy encima de ella, de los estudios (Mujer, clase obrera, rendimiento filial medio).
}

En suma, las familias jerarquizan los distintos elementos de la implicación escolar, colocando en los primeros peldaños las prácticas propias y deslegitimando el resto de comportamientos. Esta segmentación no sólo se da entre escuela y hogar, pues dentro de este último también hay espacio para nuevas divisiones. Una muy frecuente es la que distingue entre la vigilancia (de los horarios y ritmos de trabajo) y la ayuda efectiva (sentándose al lado de los hijos para explicar las tareas o hacerlas juntos). Aquí encontramos diferencias discursivas en las que, nuevamente, desempeñan un papel esencial la posición de clase y el rendimiento filial. En familias en que el capital cultural es bajo y los hijos han tenido una trayectoria tortuosa, son las técnicas de control las que se priorizan y valoran. La distancia con el universo escolar dificulta la instrucción directa, y los hijos se resisten a aceptarla al intuir reproches y discusiones. En estas circunstancias, los padres evitan "inmiscuirse" en los contenidos de la enseñanza. El apoyo escolar se expresa habitualmente fijando un tiempo mínimo de estudio y regulando todo aquello que pueda apartar a los hijos de las tareas: privación de bienes como consolas, reducción del tiempo de televisión (o de acceso a internet), limitación de los momentos de ocio con el grupo de pares, etc. Una expresión recurrente en estas posiciones, relacionada con la tensión entre autonomía e implicación es la de "ayudar a organizarse". La ayuda familiar construiría las condiciones necesarias para el aprendizaje, pero éste sería responsabilidad última de los hijos.

En el polo opuesto están las familias de clase media con hijos que no tienen dificultades especiales. Aquí la ayuda con las tareas se presenta como un componente natural de la crianza, llena de connotaciones positivas: reforzaría la relación paterno-filial, incluso permitiría a los progenitores revivir con nostalgia un agradable pasado escolar. Estos padres asumen un papel activo en la enseñanza, vinculando el apoyo a la escolaridad con la actividad propiamente formativa: identifican los conceptos importantes, organizan los contenidos e incluso tratan de ampliar los conocimientos escolares. La implicación se relaciona con sus componentes más claramente pedagógicos, mientras se degradan simbólicamente las "autoritarias" labores de inspección:

\begin{abstract}
Porque hay que dejarle su autonomía, pero seguimos, aunque no hay que ponerse a decirle "ponte a hacer las tareas", no, él coge y automáticamente, coge, se va y se poner a hacer las cosas (...) Bueno, lo típico: "pregúntame la lección" o "este ejercicio no lo entiendo" claro, te sentabas con él, lógicamente... más que dirigir el aprendizaje, no hay que dirigir, sino ayudarle en el aprendizaje, apoyarlo... y estar seguro de que lo entiende todo, porque ya el trabajo es suyo (Hombre, clase media, rendimiento filial medio).
\end{abstract}

Esta separación entre ayuda y control constituye otro eje en torno al cual los progenitores realizan multitud de jugadas discursivas. Nuevamente, son las reuniones de tutoría donde la necesidad de legitimar las prácticas familiares se impone con mayor intensidad. Excepto en casos de rendimientos excelentes, el profesorado entiende que la simple vigilancia es una inversión insuficiente. Pero, como hemos visto, son los problemas escolares filiales los que empujan a los padres a emplear métodos de control más distantes. Ello es causa de una contradicción recurrente: el profesorado demanda más ayuda efectiva cuando las condiciones de proporcionarla son más adversas para la familia:

Padre - "Porque él no hace otra cosa, le hemos quitado el móvil, vamos (...) mínimo tres horas echa todos los días que está en el cuarto y sólo tiene los libros..."

Profesora - "Que hay que vigilarle, vigilarle no es... en fin, que hay que estar pendiente, porque él se dispersa muchísimo, no está concentrado, y a lo mejor echa alli tres horas pero no ha aprovechado ni una..." (Tutoría, pareja de clase obrera, rendimiento filial bajo). 
Por tanto, la mayoría de las familias tratan de situarse en una posición más cercana a la ayuda efectiva, pero distanciándose - nuevamente- de los discursos que podrían categorizarlos como padres "asistencialistas". Para sortear esta tensión, los argumentarios parentales dibujan una amplia gama de comportamientos "intermedios", que, evitando ambos extremos, aunarían las dos legitimidades discrepantes. Se presentan entonces una serie de prácticas auxiliares, a caballo entre el control indirecto y la instrucción directa. Así, muchos progenitores enfatizan el hecho de que buscan con sus hijos conceptos en el diccionario o en internet, les ayudan a hacer esquemas, les toman la lección después de estudiar o se aseguran de que los deberes están hechos -hipotéticamente, sin hacer los deberes con ellos en ningún caso. La categorización de este adecuado punto medio es muy variada y está relacionada con los recursos parentales, el tiempo disponible y los requerimientos filiales.

\section{A la búsqueda de una legitimidad alternativa}

Hasta aquí hemos visto que la ambigüedad del concepto implicación, así como la existencia de dos polos legítimos opuestos, concede a las familias amplias posibilidades para justificar simbólicamente sus prácticas. No obstante, también hay casos en los que las estrategias parentales escapan completamente a los dictados especialistas, lo que coloca a los progenitores en una situación muy delicada, al no poder apoyar sus argumentaciones sobre las tesis dominantes.

Estas maniobras discursivas alternativas suelen descansar sobre el afecto a los hijos. La fuerza de la relación paterno-filial $-\mathrm{y}$, especialmente, maternofilial- justificaría ciertas prácticas que se alejan de las concepciones legítimas. La escuela es importante, pero más importante sería mantener la armonía de las relaciones familiares. Este discurso es característico de familias de clases populares que han aceptado un irreversible fracaso escolar filial. Los malos resultados, que aparecen tempranamente, impiden enarbolar la bandera de la autonomía infantil. El apoyo parental pasado no obtuvo los frutos deseados y los hijos muestran ahora una resistencia tenaz a la intervención paterna, que, de intentarse, desencadena conflictos en el hogar y reprobación de los docentes en la escuela. Esta dinámica lleva a los progenitores a una desconexión paulatina de la escolaridad, justificada sobre la preservación de un orden moral familiar: "yo no martirizo a mis hijos". Abandonado el compromiso con el modo de socialización escolar, el único refugio discursivo legítimo es el amor hacia los hijos:

Tampoco le puedo yo exigir mucho, no le exijo mucho, yo si me trae dos cates le digo: "nene, tienes que ponerte las pilas" pero tampoco lo castigo en... ni le quito nada de regalos ni nada porque no lo veo... que él pone de su parte, pero si él no puede más, de donde hay no puedo coger... que no lo veo, vamos
(...) Y mi amiga: "pues castígalo, no te los lleves a la playa..." Mira, yo soy incapaz, incapaz de irme yo a la playa y dejar a mi hijo aquí. (Mujer, clase obrera, rendimiento filial bajo).

En algunos casos, este amor a los hijos se expresaría a través de otro tipo de sacrificios ajenos al ámbito escolar. Como se señaló anteriormente, las familias de clase obrera que disponen de tiempo escaso -ambos cónyuges trabajan a jornada completason las que más impugnan el nuevo modo de relación escolar: éste sería propio de progenitores ociosos. Vimos que estos discursos descansan sobre la legitimidad de la autonomía filial, pero cuando el rendimiento es bajo, tales explicaciones son peliagudas, las inculpaciones de dimisión parental planean sobre la familia. Se construye entonces una legitimidad alternativa. La buena crianza no se fundamentaría en el grado de inversión pedagógica, sino en el trabajo asalariado fuera del hogar, que se contrapone a aquella. Los hijos pueden dedicarse tranquilamente a estudiar porque los padres estarían sacando a la familia adelante. De ahí que los progenitores, en estas circunstancias, tiendan a presentarse, fundamentalmente, como proveedores materiales:

Profesora - (...) Y teniendo en cuenta que vienen del verano, la primera prueba, de Septiembre, pues bueno, estaba bien, aceptablemente bien..

Padre - Claro, que nosotros en verano le hemos llevado a sitios, de museos y eso, y le hemos comprado muchos libros, todos sus libros y sus cosas (Tutoría, pareja de clase obrera, rendimiento filial bajo)

Estas posiciones cuestionan, de forma más o menos implícita, la relación entre implicación y rendimiento. Los niños serían seres muy complejos, nadie asegura que, haciéndolo todo bien, salgan estudiosos. Incapaces de encajar sus prácticas con los diversos discursos especialistas, estas familias aluden a numerosos factores externos que incidirían en las calificaciones de los hijos, sean profesores incompetentes, malas compañías, nuevas tecnologías o un innato desinterés filial. Frente a estos poderosos elementos fuera de control parental, ni siquiera un adecuado apoyo sería suficiente. Conscientes de su posición inferior en la jerarquía escolar de familias, tratan de relativizar la misma: "en el fondo, nada serviría para nada."

\section{Conclusiones}

Durante las últimas décadas hemos asistido, en España y los países de nuestro entorno, a la consolidación del discurso sobre las bondades de la relación familia-escuela. Es innegable que las disposiciones familiares frente a la educación formal han variado sustancialmente: la intensidad de la apuesta escolar ha modificado prácticas de crianza e incrementado los comportamientos de apoyo parental (Reay, 2005). No obstante, entre los promotores de 
este nuevo modelo son habituales los lamentos. Los vínculos entre familias y escuelas siempre serían imperfectos, las reformas siempre insuficientes, las relaciones siempre asimétricas.

Las intervenciones en este sentido suelen compartir un presupuesto común: la supuesta debilidad de los lazos entre escuela y hogar estaría en relación con la falta de consciencia sobre sus beneficios. Habría que enseñar a las familias -a través de tutorías o mediante Escuelas de Padres- lo importante que es ayudar. Incluso habría que enseñar a los profesores para que enseñen a las familias lo importante que es ayudar. Así, son numerosas las quejas acerca de que las relaciones con las familias ocupan un papel relegado en la formación del cuerpo docente.

Los resultados de esta investigación llevan a conclusiones distintas. En línea con otros autores (Reay, 2005; Lareau y Shumar, 1996), encontramos que las prácticas de apoyo parental guardan relación con los recursos disponibles, y que la expansión de los nuevos discursos pedagógicos no modifica la distribución de los mismos. Son las constricciones estructurales y los resultados de la trayectoria filial las que explican las estrategias parentales. Estas estrategias son habitualmente analizadas como "buenas" o "malas" en función de su adecuación a los postulados escolares, sin prestar atención a su lógica práctica.

Además, el análisis de los razonamientos parentales en torno a la escolaridad filial pone de relieve un aspecto habitualmente ignorado por la literatura: el papel activo de los hijos en la configuración de sus prácticas. Obviando esta influencia, muchos estudios sobre implicación han podido errar en sus atribuciones de causalidad. Un buen ejemplo de ello es la asociación, habitualmente señalada, entre bajo rendimiento y distanciamiento parental de la escolaridad. Nuestros resultados apuntan a que esta última es más consecuencia que causa de la primera. Estos planteamientos tienen repercusiones enormes para las investigaciones futuras, dada la conocida relación entre clase social y éxito académico. Muchas diferencias en los comportamientos de apoyo escolar, imputadas de forma directa al estatus social familiar, serían más bien producto de la desigualdad de trayectorias filiales probables (Martín Criado y Gómez Bueno, 2017b; Cooper y Crosnoe, 2007).

Pero con los análisis precedentes queremos ir más allá. Las genéricas "llamadas a la implicación" no suelen tener los resultados deseados, ya que los sujetos reinterpretan constantemente los mensajes difundidos por el sistema educativo y sus especialistas. Como acabamos de ver, las familias seleccionan tácticamente las prácticas a su alcance para satisfacer los postulados escolares, mientras desvalorizan o ignoran el resto de comportamientos recomendados. Padres y madres aprovechan la ambigüedad del concepto de implicación para negociar su sentido, ajustándolo al universo de estrategias posibles. Además, la existencia de un discurso pedagógico afianzado y legítimo —que ensalza la autonomía filial- permite a los progenitores validar su grado de inversión escolar cuando éste es inferior al teóricamente aceptable.

No sorprenden, entonces, los hallazgos de quienes han analizado el funcionamiento de los programas públicos destinados a incrementar los niveles de implicación parental. Estos han demostrado sistemáticamente su ineficacia (Mattingly et al., 2002; Zellman y Waterman, 1998; Fine, 1993,). A la luz de estos resultados, la explicación parece sencilla. Moralizar a las familias no transforma radicalmente sus comportamientos, porque los discursos especialistas son simplemente adaptados para que legitimen las disposiciones propias, y estas disposiciones están ligadas a una multiplicidad de factores materiales y sociales sobre los que no se está actuando.

\section{Agradecimientos}

El presente trabajo forma parte de una tesis doctoral en curso financiada por el programa de Formación del Profesorado Universitario (FPU). Agradezco los comentarios al diseño realizados por los miembros de mi grupo de investigación.

\section{NOTAS}

[1] El cuerpo docente, por su parte, tiende a inferir la intensidad de la implicación parental "global" a partir de las visitas de la familia al centro (Desimone, 1999) y la actitud y el rendimiento del menor (Rujas, 2016).

[2] El capital escolar es un buen ejemplo de ello. En los aspectos dependientes de éste, los sujetos más escolarizados (estudios medios) incluidos en la submuestra de clase obrera se sitúan en una posición intermedia entre sus homólogos menos cualificados y los participantes de clase media.

[3] Esta investigación utiliza el contenido de estos encuentros como material auxiliar y con el objetivo de estudiar posiciones ante la escolaridad. Un análisis por- menorizado de su funcionamiento sería el objeto de una investigación aparte (véase Weininger y Lareau, 2003).

[4] Esta tendencia general de los discursos es particularmente relevante en el caso que nos ocupa, pues estamos analizando discursos sobre la escuela frente a un interlocutor específico: un investigador/profesor universitario, agente del sistema educativo e hipotético representante de su legitimidad.

[5] Queda fuera de las pretensiones de este artículo analizar la feminización de las labores de implicación parental. Nos remitimos a este respecto al trabajo de Diane Reay (1998; 2005). 


\section{REFERENCIAS BiBLIOGRÁfiCAS}

Abouchaar, A. 2016. "Relationship between Parental Involvement, Parental Support and Family Education on Pupil Achievement and Adjustment in Schools". Journal of Changde Teachers University [Special issue]:14-27.

Alonso Carmona, C. 2014. "Familia, escuela y clase social. Sobre los efectos perversos de la implicación familiar". Revista de la Asociación de Sociología de la Educación 7(2): 395-409.

Auerbach, S. 2007. "From Moral Supporters to Struggling Advocates. Reconceptualizing parent roles in education through the experience of working-class families of color". Urban Education 42(3): 250-283. https://doi. org/10.1177/0042085907300433

Baranger, D. 2004. Epistemología y metodología en la obra de Pierre Bourdieu. Buenos Aires: Prometeo Libros.

Bennet, P. R., A. C. Lutz y L. Jayaram. 2012. "Beyond the Schoolyard: The Role of Parenting Logics, Financial Resources and Social Institutions in the Social Class Gap in Structured Activity Participation". Sociology of Education 85(2): 131-157. https://doi. org/10.1177/0038040711431585

Bower, H. A. y D. Griffin. 2011. "Can the Epstein Model of Parental Involvement Work in a High-Minority, High-Poverty Elementary School? A Case Study". Professional School Counselling 15(2): 77-87.

Bronstein, P., G. S. Ginsburg e I. S. Herrera. 2005. "Parental predictors of motivational orientation in early adolescence: A longitudinal study". Journal of Youth and Adolescence 34(6): 559-575. https://doi.org/10.1007/ s10964-005-8946-0

Cabrera, B., L. Cabrera, C. Pérez y B. Zamora. 2011. "La desigualdad legítima de la escuela justa". Revista de la Asociación de Sociología de la Educación 4(3): 307-335.

Collet-Sabé, J., X. Besalú, J. Feu y A. Tort. 2014. "Escuelas, familias y resultados académicos. Un nuevo modelo de análisis de las relaciones entre docentes y progenitores para el éxito de todo el alumnado". Profesorado: Revista de curriculum y formación del profesorado 18(2): 7-33.

Cooper, C. E. y R. Crosnoe. 2007. "The Engagement in Schooling of economically disadvantaged Parents and Children". Youth and Society 38(3): 372-391. https:// doi.org/10.1177/0044118X06289999

Cooper, K. y K. Stewart. 2017. Does Money affect children outcomes? A systematic review. York: Joseph Rowntree Foundation.

Crozier, G. 1999. "Is it a case of We Know When We're Not Wanted? The parent perspective on parent-teacher roles and relationships". Educational Research 41(3): 315328. http://dx.doi.org/10.1080/0013188990410306

Desimone, L. 1999. "Linking parent involvement with student achievement: Do race and income matter?". The Journal of Educational Research 93(1): 11-30. https://doi. org/10.1080/00220679909597625

Diamond, J. B. y K. Gomez. 2004. "African American parents educational orientations: the importance of social class and parents perceptions of schools". Education and Urban Society 36(4): 383-427. https://doi. org/10.1177/0013124504266827

Domina, T. 2005. "Levelling the Home Advantage: Assessing the effectiveness of Parental Involvement in Elemen- tary School". Sociology of Education 78(3): 233-249. https://doi.org/10.1177/003804070507800303

Egido, I. 2015. "Marcos normativos de la participación de las familias en los sistemas educativos europeos. Una visión comparada". Pp. 35-36 en La participación de las familias en la educación escolar, editado por el Consejo Escolar del Estado. Madrid: Ministerio de Educación, Cultura y Deporte.

Fine, M. 1993. "[Ap] parent involvement: Reflections on parents, power and urban public schools". Teacher College Record 94: 682-710.

Frías de Val, A. S. 2015. "Evolución del marco normativo español sobre la participación de las familias. Contexto histórico". Pp. 57-80 en La participación de las familias en la educación escolar, editado por el Consejo Escolar del Estado. Madrid: Ministerio de Educación, Cultura y Deporte.

Gillies, V. 2005. "Raising the 'Meritocracy': Parenting and the individualization of Social Class". Sociology 39(5): 835-853. https://doi.org/10.1177/0038038505058368

Goffman, E. 1971. Relations in Public. Nueva York: Basics Books.

Goldenberg, C., R. Gallimore, L. Reese y H. Garnier. 2001. “Cause or effect? Longitudinal study of inmmigrant Latino parents". American Educational Research Journal 38(3): 547-582. https://doi.org/10.3102/00028312038003547

Hill, N. E. y D. F. Tyson. 2009. "Parental involvement in Middle School: A Meta-Analytic assesment of the strategies that promote achievement". Developmental Psychology 45(3): 740-763. https://doi.org/10.1037/a0015362

Hughes, J. N., O. Kwok, y M. Hee. 2013. "Effect of retention in Firts Grade on Parents' Educational Expectations and Children's Academic Outcomes". American Educational Research Journal 50(6): 1336-1359. https://doi. org/10.3102/0002831213490784

Lareau, A. 2000. Home Advantage. Oxford: Rowman \& Littlefield Publishers.

Lareau, A. 2011. Unequal Childhoods. Berkeley: University of California Press.

Lareau, A. y W. Shumar. 1996. "The Problem of individualism in Family-School Policies". Sociology of Education 69(s): 24-39. https://doi.org/10.2307/3108454

Lee, J. y N. K. Bowen. 2006. "Parent Involvement, Cultural Capital and the Achievement Gap among Elementary School children". American Educational Research Journal 43(2): 193-218. https://doi.org/10.2307/3108454

Llin Vañó, E., C. Renau Escrig, S. Rosello Sempere, G. Marande Perrin y F. J. García Bacete. 2017. "La implicación parental según los estilos educativos y el género de los padres". Agora de Salut 4: 197-206. http://dx.doi. org/10.6035/AgoraSalut.2017.4.20.

Lott, B. 2001. "Low-income parents and the public schools". Journal of Social Issues 57(2): 247-259. https://doi. org/10.1111/0022-4537.00211

Martín Criado, E. 1991. "Del sentido como producción: Elementos para un análisis sociológico del discurso". Pp. 187-212 en El plurarismo metodológico en la investigación social: Ensayos típicos, editado por M. Latiesa. Universidad de Granada.

Martín Criado, E. 2014. "Mentiras, inconsistencias y ambivalencias. Teorías de la acción y análisis de discurso". Revista Internacional de Sociología 72(1): 115-138. 
Martín Criado, E., C. Gómez Bueno, F. Fernández Palomares y A. Rodríguez Monge. 2000. Familias de clase obrera y escuela. Donostia: Iralka.

Martín Criado, E. y C. Gómez Bueno. 2017a. "Las expectativas parentales no explican el rendimiento escolar". Revista Española de Sociología 26(1): 1-20. http:// dx.doi.org/10.22325/fes/res.2016.2

Martín Criado, E. y C. Gómez Bueno. 2017b. "El mito de la dimisión parental. Implicación familiar, desigualdad social y éxito escolar". Cuaderno de Relaciones Laborales 35(2): 305-325. http://dx.doi.org/10.5209/CRLA.56777

Martín Gimeno, R. y C. Bruquetas Callejo. 2014. "La evolución de la importancia del capital escolar en la clase obrera". Revista de la Asociación de Sociología de la Educación 7(2): 373-394.

Mattingly, D. J., R. Prislin, T. L. McKenzie, J. L. Rodriguez y B. Kayzar. 2002. "Evaluating Evaluations: The Case of Parent Involvement programs". Review of Educational Research 72(4): 549-576. https://doi. org/10.3102/00346543072004549

McGhee, E. y B. Schneider. 2009. "Parent Surveillance in Schools: A Question of social class". American Journal of Education 115(2): 195-225. https://doi. org/10.1086/595665

McGrath, D. J. y P. J. Kuriloff. 1999. "They're going to tear the doors off this place: Upper-Middle Class Parent School Involvement and the Educational Opportunities of Other People's Children". Educational Policy 13(5): 603-629. https://doi.org/10.1177/0895904899013005001

Mitchell, J. C. 1983. "Case and Situation Analysis". Sociological Review 31(2): 187-211.

Pérez Sánchez, N., M. Betancort Montesinos y L. Cabrera Rodríguez. 2014. "Inversión pedagógica y éxito escolar del alumnado de clase obrera". Revista de la Asociación de Sociología de la Educación 7(2): 410-428.

Prins, E. y B. W. Toso. 2008. "Defining and Measuring Parenting for educational success: A critical discourse analysis of the Parent Education Profile". American Educational Research Journal 45(3): 555-596. https:// doi.org/10.3102/0002831208316205
Quadlin, N. Y. 2015. “When children affect parents: Children's academic performance and parental investment". Social Science Research 52: 671-685. https://doi. org/10.1016/j.ssresearch.2014.10.007

Reay, D. 1998. Class work: Mothers' involvement in their children primary schooling. Londres: Taylor \& Francis.

Reay, D. 2001. "Finding or losing yourself?: working class relationships to education". Journal of Education policy 16(4): 333-346. https://doi. org/10.1080/02680930110054335

Reay, D. 2005. "Doing the dirty work of social class? Mothers' work in support of their children's schooling". The Sociological Review 53(s2): 104-116. https://doi. org/10.1111/j.1467-954X.2005.00575.x

Rujas Martínez-Novillo, J. 2016. "Cómo juzga la escuela a las familias". Revista de la Asociación de Sociología de la Educación 9(3): 385-396.

Small, M. L. 2008. "Lost in translation: How not to make qualitative research more scientific". Pp. 165-172 en Report from workshop on interdisciplinary standards for systematic qualitative research, editado por Lamont, M., \& White, P. Washington DC: National Science Foundation.

Tarabini, A. 2015. "La meritocracia en la mente del profesorado: Un análisis de los discursos docentes en relación al éxito, fracaso y abandono escolar". Revista de la Asociación de Sociología de la Educación 8(3): 349360.

Vincent C. y C. Maxwell. 2016. "Parenting priorities and pressures: Furthering understanding of concerted cultivation". Discourse: Studies in the cultural politics of education 37(2); 269-281.

Weininger, E. B. y Lareau, A. 2003. "Translating Bourdieu into the American context: the question of social class and family-school relationships". Poetics 31(5-6): 375-402. https://doi.org/10.1016/S0304-422X(03)00034-2

Zellman, G. G. y J. M. Waterman. 1998. "Understanding the impact of Parent School Involvement on Children's Educational Outcomes". The Journal of Educational Research 91(6): 370-380. https://doi. org/10.1080/00220679809597566

CARLOS ALONSO CARMONA. Doctorando en Ciencias Sociales en la Universidad Pablo de Olavide (Sevilla). El presente trabajo forma parte de una tesis doctoral en curso financiada por el programa de Formación del Profesorado Universitario (FPU). 